garden of Eden before the serpent introduces science, and with that, the need for natural-kind concepts). Those readers prepared to stick through this small but densely argued book will be rewarded with a sparkling display of elo- quence, as well as a glimpse of the vast panorama of issues surrounding what you might have thought was a small technical problem, but which Fodor shows us is one of the central outstanding problems of cognitive science.
Zenon W. Pylyshyn

Rutgers Center for Cognitive Science, Rutgers University, New Brunswick, NJ, USA. tel: +1 9084450635 fax: +19084456715 e-mail: zenon@ruccs.rutgers.edu

\title{
The Atomic Components of Thought
}

by John R. Anderson and Christian Lebiere, Lawrence Erlbaum, 1998. \$34.95 (pbk) (xi + 490 pages) ISBN 0805828168 (hbl) / 0805828176 (pbk)

Thomas Kuhn's theory of scientific revolutions ${ }^{1}$ hasn't had a good influence on cognitive science. Kuhn depicts the world of science as having periods of normal research in which scientists stick to a fixed paradigm, alternated by revolutions in which the current scientific establishment is overthrown. Because normal research in terms of Kuhn seems rather boring, many cognitive scientists present their theories as new Kuhnian paradigms by discrediting others. Now a research community of revolutionaries might lead to exciting debates, but can prevent the synergy of ideas that are not mutually exclusive.

In 1993 Anderson $^{2}$ presented an exception: the 'ACT-R' (Adaptive Control of Thought-Rational) theory. It combines ideas from the Newell and Simon ${ }^{3}$ symbolic framework with concepts from connectionist models. ACT-R is not just a theory, it is an architecture of cognition, a platform that can be used to implement models of all kinds of cognitive tasks. These models make precise predictions about behavior: reaction times, errors, choices, down to the detail of shifting visual attention and motor control.

ACT-R has two long-term memory stores, a declarative and a procedural memory. Each of these memories has two layers of representation: a symbolic and a sub-symbolic layer. Declarative memory contains facts and goals, which are, at the symbolic level, represented by a semantic network. The subsymbolic level has many aspects in common with neural networks: each declarative fact has an activation level, which is in part dependent on activation it receives from other facts through association strengths. The activation of a fact determines if it can be retrieved from memory at a certain moment, and the time it takes to do so. If several facts are applicable at the same time, the fact with the highest activation is chosen. So, the sub-symbolic level fills in the gaps left by the symbolic level. Similar rules apply to procedural memory. All elements of representation are susceptible to change through learning: achieved goals are stored as new facts in declarative memory, examples can be generalized to new rules and all the sub-symbolic parameters are continuously updated through experience and decay in time.

The book not only explicates ACT$R$, but also shows the results of five years of modeling. These models are an important source of evidence for the theory, since they provide experimental support for separate aspects of the theory, and for the theory as a whole. The first four chapters of the book explain ACT-R in detail. The nice thing about this explanation is that each detail of the theory is supported by a model. For example, the mechanism that takes care of maintaining the activation levels of facts in declarative memory is illustrated by a model of alphabet arithmetic: an experiment ${ }^{4}$ in which participants have to check expressions like $A+3=D$. According to the ACT-R model, there are two ways to do this: by counting down the alphabet, or by retrieving the fact from memory. Becauses people usually do not have facts of alphabet arithmetic in memory, participants in the experiment and the ACT-R model both start with counting as their strategy. However, once they get some experience, they might retrieve an answer from memory instead, and they become faster as the same example is repeated more often. Both the shift in strategy and the subsequent decrease in reaction time are predicted perfectly by the model.

After introducing the theory, the two subsequent chapters outline how ACT-R communicates with the outside world using modules for visual perception, motor control, etc. Again, the proposed structures are supported by ample models. The rest of the book is organized around some major themes in cognitive science: list memory, choice, cognitive arithmetic, analogy and scientific discovery. In each of these domains it is shown how deeper understanding can be gained by explaining (and modeling) the most important findings in the domain in terms of the ACT-R theory.

ACT-R brings cognitive science back to the essence of science; that is, to find theories that can make precise predic tions, and that are general enough to be applicable in many domains and

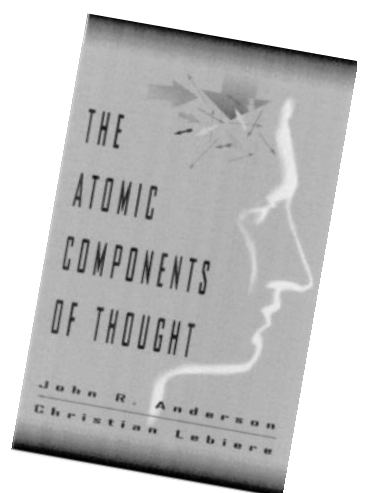

situations. A possible criticism of the theory is that it lacks parsimony - that is has too many mechanisms, too much freedom in representation and too many parameters to tweak. The authors are aware of this problem, and try to find fixed values for parameters and restrictions on the representation of production rules in order to strengthen the theory. Still, ACT-R is a theory in development, as witnessed by the final chapter of the book. A website (http://act.psy.cmu.edu) further supports the book, containing an extensive ACT-R tutorial and the opportunity to download all the software and models in the book. It is even possible to run the models on-line, and see how the outcome of a model changes due to changes in parameters.

This is in an important book for everyone interested in the simulation of human intelligent behavior. It puts forward a compelling theory that provides hope that unification in cognitive science is not lost, and it could rescue cognitive science from Kuhnian chaos.

\section{Niels Taatgen}

Department of Cognitive Science and

Engineering, University of Groningen, Grote Kruisstraat 2/1, 9712 TS Groningen,

The Netherlands.

tel: +31503636435

fax: +31503636304

e-mail: n.a.taatgen@bcn.rug.nl

\section{References}

1 Kuhn, T.S. (1970) The Structure Of Scientific Revolutions, University of Chicago Press

2 Anderson, J.R. (1993) Rules Of The Mind Lawrence Erlbaum

3 Newell, A. and Simon, H.A. (1972) Human Problem Solving, Prentice-Hall

4 Zbrodoff, N.J. (1995) Why is $9+7$ harder than $2+3$ ? Strength and interference as explanation of the problem-size effect Mem. Cognit. 6, 689-700 